tits / C

NHGW -4639

Force balance and substorm effects in the magnetotail NAG5-4453

Richard L. Kaufmann, Douglas J. Larson, Ioannis D. Kontodinas, and Bryan M. Ball

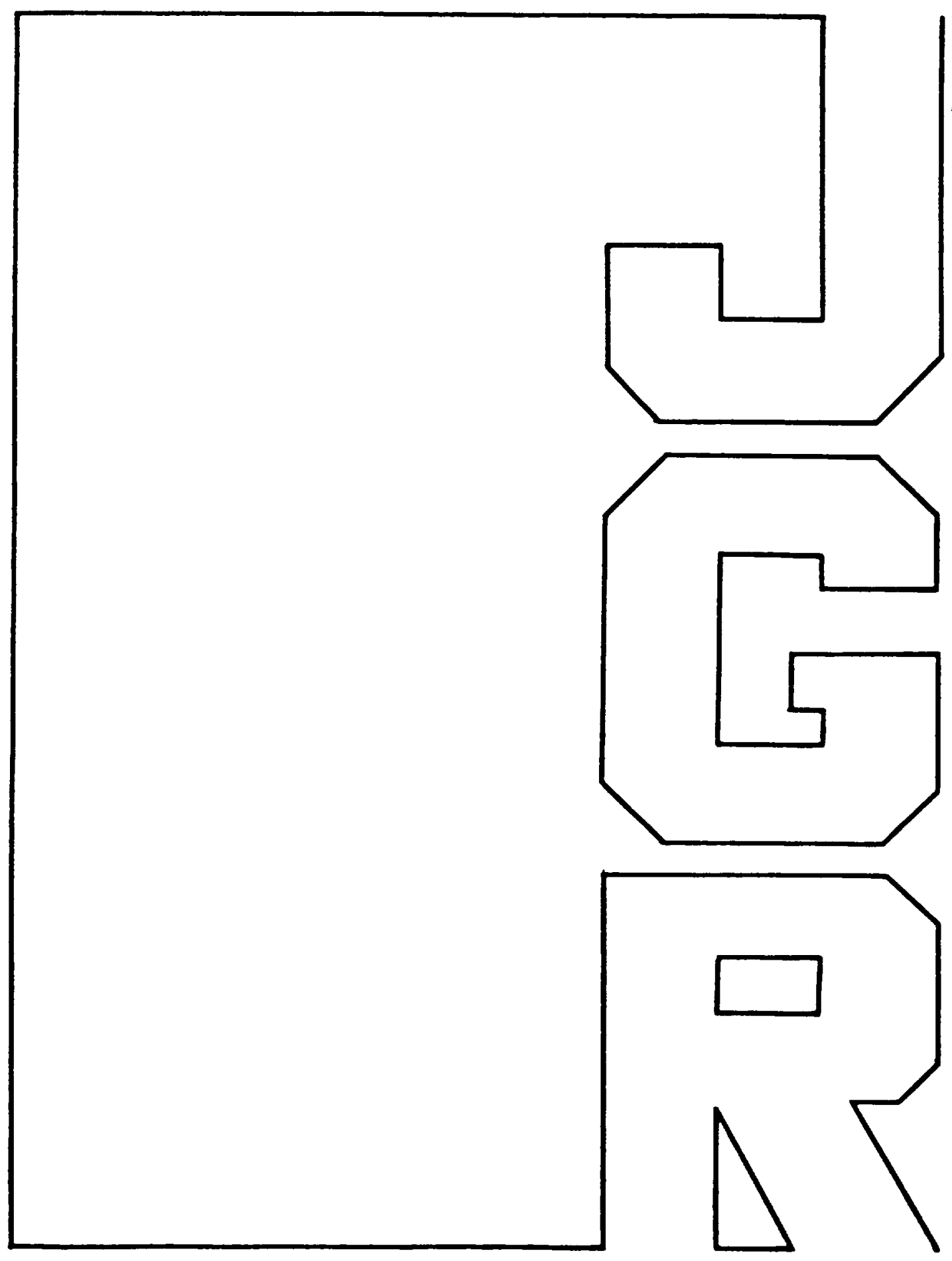

Journal of Geophysical Research, vol. 102, no. A 10, Oct. 1, 1997 


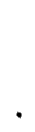




\title{
Force balance and substorm effects in the magnetotail
}

\author{
Richard L. Kaufmann, Douglas J. Larson, Ioannis D. Kontodinas, and Bryan M. Ball \\ Department of Physics, University of New Hampshire, Durham
}

\begin{abstract}
A model of the quiet time middle magnetotail is developed using a consistent orbit tracing technique. The momentum equation is used to calculate geocentric solar magnetospheric components of the particle and electromagnetic forces throughout the current sheet. Ions generate the dominant $x$ and $z$ force components. Electron and ion forces almost cancel in the $y$ direction because the two species drift earthward at comparable speeds. The force viewpoint is applied to a study of some substorm processes. Generation of the rapid flows seen during substorm injection and bursty bulk flow events implies substantial force imbalances. The formation of a substorm diversion loop is one cause of changes in the magnetic field and therefore in the electromagnetic force. It is found that larger forces are produced when the cross-tail current is diverted to the ionosphere than would be produced if the entire tail current system simply decreased. Plasma is accelerated while the forces are unbalanced resulting in field lines within a diversion loop becoming more dipolar. Field lines become more stretched and the plasma sheet becomes thinner outside a diversion loop. Mechanisms that require thin current sheets to produce current disruption then can create additional diversion loops in the newly thinned regions. This process may be important during multiple expansion substorms and in differentiating pseudoexpansions from full substorms. It is found that the tail field model used here can be generated by a variety of particle distribution functions. However, for a given energy distribution the mixture of particle mirror or reflection points is constrained by the consistency requirement. The study of uniqueness also leads to the development of a technique to select guiding center electrons that will produce charge neutrality all along a flux tube containing nonguiding center ions without the imposition of a parallel electric field.
\end{abstract}

\section{Introduction}

\subsection{Consistent Orbit Tracing Method}

The consistent orbit tracing (COT) technique [Larson and Kaufmann, 1996] (hereafter referred to as LK96) was used to construct a steady state model current sheet. In a consistent model the ions and electrons carry the electric current needed to produce the magnetic field in which their orbits were traced. The COT method begins with a preselected magnetic field and a search is made to find particles that will produce this field. In contrast, most simulations begin with preselected particle sources and boundary conditions and calculate the resulting fields.

The standard magnetic field plus uniform cross-tail electric field model described in LK96 was used here. The magnetic field was adjusted so that the adiabaticity parameter defined by $\kappa^{2}=$ $R_{\min } / \rho_{\max }$ [Büchner and Zelenyi, 1989] and the total cross-tail sheet current density $K_{y}(x)$ in $\mathrm{A} / \mathrm{m}$, which is the volume current density $j_{y}(x, z)$ in $\mathrm{A} / \mathrm{m}^{2}$ integrated through the thickness of the current sheet, were similar to those at $y=0$ in the $K p=4$ version of the Tsyganenko [1989] or T89 model. In the above definition $R_{\min }$ is the minimum radius of curvature of a magnetic field line and $\rho_{\max }$ is the maximum gyroradius of an ion. Both $R_{\min }$ and $\rho_{\max }$ are found at $z=0$. The region studied in detail was $-20 R_{E}<x<$ $-14 R_{E}, 0<|z|<2 R_{E}$ in geocentric solar magnetospheric coordinates. The standard model current sheet has a characteristic $z$ scale

Copyright 1997 by the American Geophysical Union.

Paper number 97JA01723.

0148-0227/97/97JA-01723\$09.00 length of $0.7 R_{E}$ in this region. All the numerical calculations were fully three-dimensional (3-D). However, since our standard magnetic field model has significant $y$ dependence only near and sunward of $x=-10 R_{E}$, the model is essentially 2-D in the region of interest.

Since the COT analysis finds particles that create a model magnetic field, it is important to start with a model that is representative of the actual magnetotail. T89 is an empirical model involving many parameters that were adjusted to fit a large collection of satellite data. The data were sorted according to geomagnetic indices such as $K p$ but not according to substorm phase. As a result, the model tail represents an average over both the thin stretched configuration that is seen before a local substorm expansion and the dipolar configuration seen after expansion. It therefore is not necessary that the model will provide a good representation of the Earth's field at any one time.

One important question is whether the model is consistent with steady convection. Erickson and Wolf [1980] pointed out that adiabatic fluid convection in common magnetotail models will result in plasma pressures in the inner plasma sheet that are much higher than is observed. Some models have specifically been developed to be consistent with quasi static convection [ $\mathrm{Hau}, 1991]$. This shows that it is possible to develop a steady state magnetic field model in which forces are nearly balanced. Chen and Wolf [1993] developed a nonuniform model containing small plasma bubbles that are accelerated primarily in the distant tail and decelerated primarily in the near-Earth plasma sheet. This model was constructed so that it can explain the observed pressure profile. Finally, it is possible that the plasma sheet is usually evolving smoothly as in many MHD 
models [Walker et al., 1993; Birn et al., 1996]. In this scenario the field approaches a quasi-static configuration [ $\mathrm{Hau}, 1991]$ only during periods of steady convection [Sergeev et al., 1996a]. These topics will be discussed in more detail in a paper that is devoted to a study of the energy equation, which describes the evolution of the pressure tensor as plasma flows toward the Earth.

Groups of 1000 ions each were traced in the model fields to carry out a COT analysis. A starting energy, pitch angle, and phase angle were randomly selected for each ion using a 5-keV Maxwellian parent population. The starting $x$ and $z$ positions were randomly distributed over one of the spatial boxes. Each group used in LK96 also was composed of 1000 ions, but those ions were selected from various monoenergetic parent distribution functions. No explicit boundary conditions were imposed at the edges of the region of interest. Particles drifted from a physical source somewhere tailward of $x=-20 R_{E}$ to a sink somewhere earthward of $x=$ $-14 R_{E}$. Orbits were traced both forward and backward in time until the particle drifted so far it could never return to the region of interest.

The energy of each ion changed as the particle drifted and different groups were started at different locations. The final energy distribution in a given spatial box therefore was broader than the starting distribution. Full 3-D velocity distribution functions were retained in 120 spatial boxes for each group of ions that was traced. The boxes were $0.1-R_{E}$ wide in the $z$ direction and $1-R_{E}$ wide in the $x$ direction. The model is symmetric about $z=0$. Only one very wide box was used in the $y$ direction because no useful information would be gained by keeping multiple $y$ boxes in a region that has essentially no $y$ dependence.

Electron currents were added to the current calculated for each group of ions. A number of ion-plus-electron groups then were combined to form a plasma that approximately carries the current which is needed to produce the magnetic field. The combination of groups was done with a least squares fitting method that considered only the $y$ component of $\mathbf{j}$.

The decision to use 1000 ions for each group was made by simply increasing the number of particles per group until repeating the orbit tracing process with multiple groups which were randomly selected from the same parent population produced almost identical current patterns. Since only $j_{y}$ was used for the fitting routine, there was no advantage in increasing the size of each group at this point in the analysis. Larger groups were used for some studies, such as those which needed accurate values of off diagonal elements in the pressure tensor.

A total of 20 to 40 groups of ions usually were traced to carry out a COT analysis. However, an average of only about eight groups was kept to produce the final plasma. Keeping more groups produced better agreement between the $j_{y}$ carried by particles and the $j_{y}$ that is needed to produce the model field. However, our principal goals involve learning more about the physical structure of the current sheet rather than producing the closest possible fit to a preselected model. We therefore required that each group in the final plasma must be needed at a $95 \%$ confidence level to improve the agreement between the model $j_{y}$ and the desired $j_{y}$.

\subsection{Background, Goals, and Overview}

The advantages of the COT method are it produces approximately consistent kinetic current sheet models which satisfy the Vlasov equation; the use of very accurate orbit tracing assures that all nonguiding center aspects of particle trajectories are included; and 3-D ion velocity distribution functions $f_{i}(\mathbf{r}, \mathbf{v})$ are generated for each of the spatial boxes. The availability of $f_{i}(r, v)$ permits a detailed study of the structure of the neutral sheet region, where ion orbits are very complex. It is easy to evaluate all the fluid parameters and derivatives that are needed to calculate forces and energy flows and to make comparisons with observations.

The most important disadvantage of the COT technique is that it only generates a steady state model. The topics of stability, fluctuations, and time evolution are not addressed by these calculations. The COT results may provide good starting distributions for future kinetic simulation and instability studies. For practical reasons the present COT results covered a relatively small region of the magnetotail. The results in this paper therefore cannot be used to locate the solar wind and ionospheric sources of plasma sheet particles, as was done using the large-scale kinetic orbit tracing calculations [Peroomian and Ashour-Ahdalla, 1995]. The limited region that was modeled using the COT technique also means that it is not possible to integrate throughout a flux tube from the ionosphere to the equator. This limitation makes it hard to compare the present results to bounce averaged calculations.

Methods that are similar to the COT technique were used by Eastwood [1975]. Other early orbit tracing studies relevant to the COT technique include Speiser [1965, 1970], Alekseyev and Kropotkin [1970], Sonnerup [1971], Francfort and Pellat [1976], Cowley and Pellat [1979], Wagner et al. [1979], and Gray and Lee [1982]. The application of techniques from chaos theory by Büchner and Zelenyi [1986] and Chen and Palmadesso [1986] were particularly important. These and other early studies are described by Kaufmann and Lu [1993] and Kaufmann et al. [1994] where a version of the COT method suitable for 1-D magnetotail models was developed. The 1-D studies found that it is easy to generate COT current sheets that produce a modified Harris [1962] model magnetic field. However, the resulting current sheets were not consistent with observations by satellites in the magnetotail. The 2-D models used in LK96 produced current sheets that agreed with observations.

The first goal of the present paper is to see how accurately forces are balanced in the COT model and to calculate the relative importance of each term in the momentum equation. Average magnitudes of these terms were estimated long ago using quiet time measured plasma parameters and fields [Cole and Schindler, 1972; Rich et al., 1972]. The present paper gives a more detailed comparison using the full pressure tensor to calculate the $x$ and $z$ spatial dependence of each Cartesian component of the force terms. It also is important to check the reliability of the COT results that use derivatives of the pressure tensor. The accuracy of such derivatives must be assessed for a future study of energy flow, heat flow, entropy changes, and equations of state. Section 2 is devoted to a study of the momentum equation and an evaluation of terms derived by the COT analysis.

Events that occur near substorm onset are studied in section 3 as a demonstration of the usefulness of the force viewpoint. Substorm processes in the magnetotail usually are described in terms of the disruption of current in the near-Earth plasma sheet and the diversion of current to the ionosphere. The alternative force viewpoint emphasizes the unbalanced forces that develop near substorm onset. The fact that changes in both the local electromagnetic force and the local bulk flow velocity can be measured in data from a single satellite also may make it possible to directly observe some consequences of force imbalance. It is much more difficult to directly measure changes in a full substorm current system.

The above results are summarized and compared with recent orbit tracing and kinetic analyses in section 4 . The appendices address some questions of uniqueness. It is seen that a range of distribution functions could produce the preselected 2-D magnetic 
field. The more complex problem involving 3-D fields with strong $y$ dependence is not treated. It also is shown that the method used to add electrons and to calculate the associated parallel electric field $E_{\|}$is not unique. A technique is derived to adjust the equatorial guiding center electron pitch angle distribution to assure charge neutrality with a given nonguiding center ion density distribution and $E_{\|}=0$.

A companion paper [Kaufmann et al., this issue] considers nonguiding center aspects of the COT model. This companion study emphasizes the importance of thin current sheets and weak magnetic fields near $z=0$. Nonguiding center mechanisms that may disrupt cross-tail current in a thin current sheet without significantly decreasing the tail plasma population also are described. Future papers will be devoted to an analysis of the energy equation and a comparison of the calculated 3-D velocity distribution functions with observations.

\section{Force Balance}

\subsection{Stress Tensors}

Forces can be calculated by taking derivatives of various stress tensors. The particle stress or kinetic tensor for particles of species $s$ is [Spitzer, 1962; Rossi and Olbert. 1970]

$$
T_{s, \alpha \beta}=m_{s} \int v_{\alpha} v_{\beta} f_{s}(\mathbf{r}, \mathbf{v}) d^{3} v=P_{s, \alpha \beta}^{(s)}+\rho_{s} V_{s, \alpha} V_{s, \beta}
$$

where

$$
v_{s, \alpha}=\frac{1}{n} \int v_{\alpha} f_{s}(\mathbf{r}, \mathbf{v}) d^{3} v
$$

is the bulk flow velocity of the species

$$
P_{s, \boldsymbol{\alpha} \beta}^{(s)}=m_{s} \int\left[v_{\alpha}-V_{s, \boldsymbol{\alpha}}\right]\left[v_{\beta}-v_{s, \beta}\right] f_{s}(\mathbf{r}, \mathbf{v}) d^{3} v
$$

is the individual-pressure tensor for the species and $f_{s}(\mathbf{r}, \mathbf{v})$ is the velocity distribution function. The superscript in $\mathbf{P}_{s}^{(s)}$ indicates that the individual-pressure tensor is defined in a frame moving at the bulk flow velocity of species $s$. The above notation is similar to that used by Rossi and Olbert [1970]. In (1) - (3) $\alpha$ and $\beta$ designate Cartesian components, the particle mass is $m_{s}, n_{i}=n_{e}=n$ is the density of the singly charged ions and of electrons, $\rho_{s}=m_{s} n$ is the mass density, and $\rho_{s} V_{s, \alpha} V_{s, \beta}$ is the Reynolds stress or dynamic-pressure tensor. For an ion-electron plasma we neglect the small difference between the frame moving at the ion bulk velocity $V_{i}$ and the proper frame which moves at $\mathbf{V}$, the mass weighted ion plus electron bulk velocity defined by $\rho \mathbf{V}=m_{i} n_{i} \mathbf{V}_{i}+m_{e} n_{e} V_{e}$. This approximation gives $\mathbf{P}_{i}^{(i)} \cong \mathbf{P}_{i}$, where $\mathbf{P}_{i}$ is the usual pressure tensor, defined by (3) with $\mathbf{V}_{s}$ replaced by $\mathbf{V}$. An important property of the fluid parameters defined above is that they all depend only on the particle information contained in $f_{s}(\mathbf{r}, \mathbf{v})$. All ion fluid parameters were calculated by integrations over the COT velocity distribution functions.

The COT ion distribution functions were generated by noting that $f_{s}(\mathbf{r}, \mathbf{v})$ for any one spatial-velocity or phase space box is proportional to the total time all ions spent in the desired velocity box while the particles also were located in the spatial box of interest. For each time step along each trajectory, half the time increment was placed in the phase space box in which the particle was located at the start of the step and half into the phase space box cor- responding to the end of the time step. Velocity space was divided into 30 boxes, 15 for each positive and 15 for each negative Cartesian component of velocity. The result is a 27,000 -point 3-D velocity distribution function for each of the 120 spatial boxes.

The stress tensor $\mathbf{T}_{s}$ contains everything that is needed concerning the kinetic energy of plasma particles. The separation on the right side of $(1)$ into $\mathbf{P}_{s}^{(s)}$, which contains information about the thermal kinetic energy, and the Reynolds stress tensor, which contains bulk flow information, will be used throughout this work.

In contrast, the electromagnetic stress tensor depends only upon the model electric and magnetic fields [Jackson, 1975, p. 239]

$$
T_{\alpha \beta}^{E M}=\varepsilon_{o} E_{\alpha} E_{\beta}+\frac{1}{\mu_{o}} B_{\alpha} B_{\beta}-\frac{1}{2}\left[\varepsilon_{o} \mathbf{E} \cdot \mathbf{E}+\frac{1}{\mu_{o}} \mathbf{B} \cdot \mathbf{B}\right] \delta_{\alpha \beta}
$$

Forces produced by the particles whose orbits were traced to generate the model current sheet will be compared with forces exerted by the preselected fields.

\subsection{Force Equations}

A model current sheet generated by the COT technique ideally results in force balance. This can be seen by noting that the Vlasov equation is based on the assumption that each particle follows an unperturbed orbit in the average $\mathbf{B}$ and $\mathbf{E}$ fields produced by all other ions and electrons. The COT technique explicitly follows such unperturbed orbits. This method therefore is equivalent to solving the Vlasov equation provided the final plasma sheet is consistent in the sense described above. The momentum equation is derived by taking the first velocity moment of the Vlasov equation. For nonrelativistic particles with charge $q_{s}$ and neglecting gravity this gives [Spitzer, 1962; Rossi and Olbert, 1970]

$$
\begin{gathered}
n_{s} m_{s} \frac{\partial V_{s, \alpha}}{\partial t}+n_{s} m_{s}\left(\mathbf{V}_{s} \cdot \nabla\right) V_{s, \alpha}+\sum_{\beta=1}^{3} \frac{\partial P_{s, \beta \alpha}^{(s)}}{\partial x_{\beta}} \\
=n_{s} q_{s} E_{\alpha}+\left(\mathbf{j}_{s} \times \mathbf{B}\right)_{\alpha}
\end{gathered}
$$

The left side of (5) depends only on the particle kinetic energy terms defined in (1) - (3). The right side of (5) contains all information about the fields and about the fact that a plasma is composed of charged particles rather than neutral atoms. The sum in (5) is the divergence of the individual-pressure tensor $\nabla \cdot \mathbf{P}_{s}^{(s)}$. The momentum equation describes changes in the bulk flow velocity that are produced by force imbalances. The next velocity moment of the Vlasov equation gives the energy equation, which will be investigated in a separate paper. The energy equation is used to study changes in the pressure tensor as a result of energy and heat fluxes that flow into and out of a given box. As noted previously, the energy equation is needed to study topics such as the pressure balance inconsistency [Erickson and Wolf, 1980; Hau, 1991; Erickson, 1992; Ashour-Abdalla et al., 1994].

Ion and electron equations (5) are added to get a plasma momentum equation. When adding ion and electron terms it is assumed that the magnetotail is charge neutral and that terms of order $m_{e} / m_{i}$ can be neglected relative to unity, e.g. the mass density is $\rho=m_{i} n_{i}+m_{e} n_{e} \cong m_{i} n$. Electrons in the tail are observed to have about $1 / 7$ the energy of ions [Baumjohann et al., 1989], so that the bulk drift velocities satisfy $V_{e}<V_{i}$ in a quiet time approximately $1-R_{E}$ thick tail even though the total particle velocities satisfy $v_{e} \gg v_{i}$ (LK96). Electron drift currents can be more important 
in thinner tails. The above approximations show that electron effects are significant only in the $\mathbf{P}$ and $\mathbf{j}$ terms of (5) yielding

$$
\rho \frac{\partial V_{\alpha}}{\partial t}+\rho(\mathbf{V} \cdot \nabla) V_{\alpha}+\sum_{\beta=1}^{3} \frac{\partial P_{\beta \alpha}}{\partial x_{\beta}}=(\mathbf{j} \times \mathbf{B})_{\alpha}
$$

where $\mathbf{j}=\mathbf{j}_{c}+\mathbf{j}_{i}$; and $P_{\beta \alpha}=P_{e, \beta \alpha}+P_{i, \beta \alpha}$. As noted previously, with the above approximations the ion frame is the same as the proper frame which moves at velocity $\mathbf{V}$. Electric field forces have cancelled in the neutral plasma. The $\partial V_{\alpha} / \partial t$ factor in (6) is the acceleration of plasma as seen by a satellite, since satellites in the magnetotail are essentially stationary. The first two terms in (6) are often combined to give $\rho d V_{\alpha} / d t$, where $d V_{\alpha} / d t$ is the acceleration of a moving element of the plasma. The second term in (6) is nonzero even in a steady state model because the convection speed changes as each plasma element flows in from $-20 R_{E}$ to $-14 R_{E}$ (LK96). It again is seen that the ion plus electron momentum equation, which follows directly from the Vlasov equation, relates particle terms on the left side of (6) to particle and field terms on the right side of $(6)$.

It sometimes is useful to think of each individual ion group as a separate species with $s$ being the group number. There then would be many ion species with the same mass and charge. Equations (5) and (6) were used with separate groups of 1000 ions plus electrons to check the COT analysis. When using (6) with a single group $\mathbf{V}$ is the bulk velocity of that group, $\mathbf{P}$ is the partial pressure associated with that group as defined in the group's frame, and $\mathbf{j}$ is the partial current carried by particles in the group. Only $\mathbf{B}$ involves the combination of currents from all particles.

Kinetic tensors $\mathbf{T}_{s}$ can be added since they are all defined in the Earth frame. It is not appropriate to weight and add individual-pressure tensors when combining the separate groups to form a consistent plasma because the $\mathbf{P}_{s}^{(s)}$ for each group was defined in a different reference frame. The method used to combine groups here and in LK96 was to weight and sum the ion group distribution functions. This produced a single ion distribution function for the consistent plasma. The final plasma fluid parameters then were calculated by integrations such as those in (1) - (3) using this combined ion distribution function.

Ampere's law can be used when (6) is applied to the full consistent model. In this case $\left(1 / \mu_{0}\right) \nabla \times \mathbf{B}-\varepsilon_{0} \partial \mathbf{E} / \partial t$ can be substituted for the combined $\mathbf{j}$ from all particle groups, where $\mathbf{B}$ and $\mathbf{E}$ always refer to the preselected fields. The magnetic field cross product can be expanded yielding the usual magnetic field pressure and tension forces. We conclude that it is possible to fully separate particle and field terms only in the final consistent model current sheet that is produced by adding contributions from all particle groups. The COT method then ideally results in balance between the particle terms on the left side of (7)

$$
\begin{aligned}
& \rho \frac{\partial V_{\alpha}}{\partial t}+\rho(\mathbf{V} \cdot \nabla) V_{\alpha}+\sum_{\beta=1}^{3} \frac{\partial P_{\beta \alpha}}{\partial x_{\beta}} \\
& =\left[\frac{1}{2 \mu_{o}} \nabla B^{2}-\frac{1}{\mu_{o}}(\mathbf{B} \cdot \nabla) \mathbf{B}\right]_{\alpha}
\end{aligned}
$$

which were calculated using only $f_{s}(r, v)$ and the terms on the right side of (7), which depend only on the preselected fields.
In this work it sometimes is useful to consider the ions and electrons separately because ions were treated through orbit tracing and electrons through the guiding center approximation. It was seen above that the electron and ion $n q \mathbf{E}$ forces cancel in a neutral plasma. Ion and electron $\mathbf{E} \times \mathbf{B}$ drift currents also cancel. To examine ions and electrons separately, it may be noted that these statements are equivalent to the observation that the portion of the $\mathbf{j} \times \mathbf{B}$ force attributable to $\mathbf{E} \times \mathbf{B}$ drift for either species exactly cancels the $n q \mathbf{E}$ force for that species, provided $\mathbf{E} \cdot \mathbf{B}=0$. One simple way to see this involves transforming to a frame in which the electric field vanishes at one point of interest, so that both $\mathbf{E} \times \mathbf{B}$ and $n q \mathbf{E}$ are zero.

\subsection{Electron Terms}

All ion quantities in (6) and (7) were calculated directly. Electrons were assumed to be isotropic and to obey the guiding center equations because the electron adiabaticity parameter $\kappa$ is much larger than one. The average ion scalar pressure $P_{i}(x, z)=$ $(1 / 3) \operatorname{Tr}\left(\boldsymbol{P}_{i}\right)$ was evaluated for each box, and a polynomial fit was made to the resulting array. The polynomial fit was used in $P_{c}(x, z)=(1 / 7) P_{i}(x, z)$ to calculate the electron pressure. The total current carried by guiding center particles

$$
\begin{aligned}
& \mathbf{j}_{s \perp}=\frac{\mathbf{B}}{B^{2}} \times\left[\nabla P_{s \perp}+\frac{P_{s l l}-P_{s \perp}}{B^{2}}(\mathbf{B} \cdot \nabla) \mathbf{B}\right] \\
& +n_{s} q_{s} \frac{\mathbf{E} \times \mathbf{B}}{B^{2}}+n_{s} m_{s} \frac{\mathbf{B}}{B^{2}} \times\left[\left(\mathbf{V}_{s} \cdot \nabla\right) \mathbf{V}_{s}\right]
\end{aligned}
$$

was used with $P_{c l l}=P_{c \perp}$ to evaluate $\mathbf{j}_{e \perp}(x, z)$. Equation (8) can be obtained by combining guiding center gradient, curvature, $\mathbf{E} \times \mathbf{B}$, polarization, and all magnetization drift terms [Parker, 1957]. Although it does not affect calculations of the $\mathbf{j} \times \mathbf{B}$ force, the electron parallel current was selected to cancel the ion $j_{11}$ because a steady state model with negligible $y$ dependence cannot support region 1 or 2 current systems. This choice of $j_{\| \mid}$also was needed to make $\nabla \cdot \mathbf{j}=0$. The last term in (8) involves polarization drift, and is negligible for electrons. Using $\mathbf{V}_{c}=\mathbf{j}_{e} / n q_{c}$ these expressions provide all the electron parameters needed in (6) and (7).

\subsection{Tests of Force Balance}

Several tests can be made to see if the model is realistic. The electromagnetic force on a unit volume of plasma is written either as

$$
F_{\alpha}^{E M}=\sum_{\beta=1}^{3} \frac{\partial T_{\beta \alpha}^{E M}}{\partial x_{\beta}}
$$

or, when $\mathbf{E}$ is uniform, as the right side of (7). The smooth solid lines in Figure I show the three Cartesian components of $F^{E M}$ evaluated using (9) and based solely on the preselected fields. The dashed lines are components of $\mathbf{j} \times \mathbf{B}$ calculated using the preselected field and currents carried by the COT model ions and electrons. The $y$ component of $\mathbf{j}(x, z)$ produces the $x$ and $z$ components of $\mathbf{j} \times \mathbf{B}$. It therefore is not surprising that the agreement between these components of $F^{E M}$ and $\mathbf{j} \times \mathbf{B}$ in Figure 1 are as good as the typical agreement between the $j_{y}(x, z)$ carried by particles in the model and the goal $j_{y}(x, z)$ that is needed to consistently generate the preselected magnetic field (LK96). 

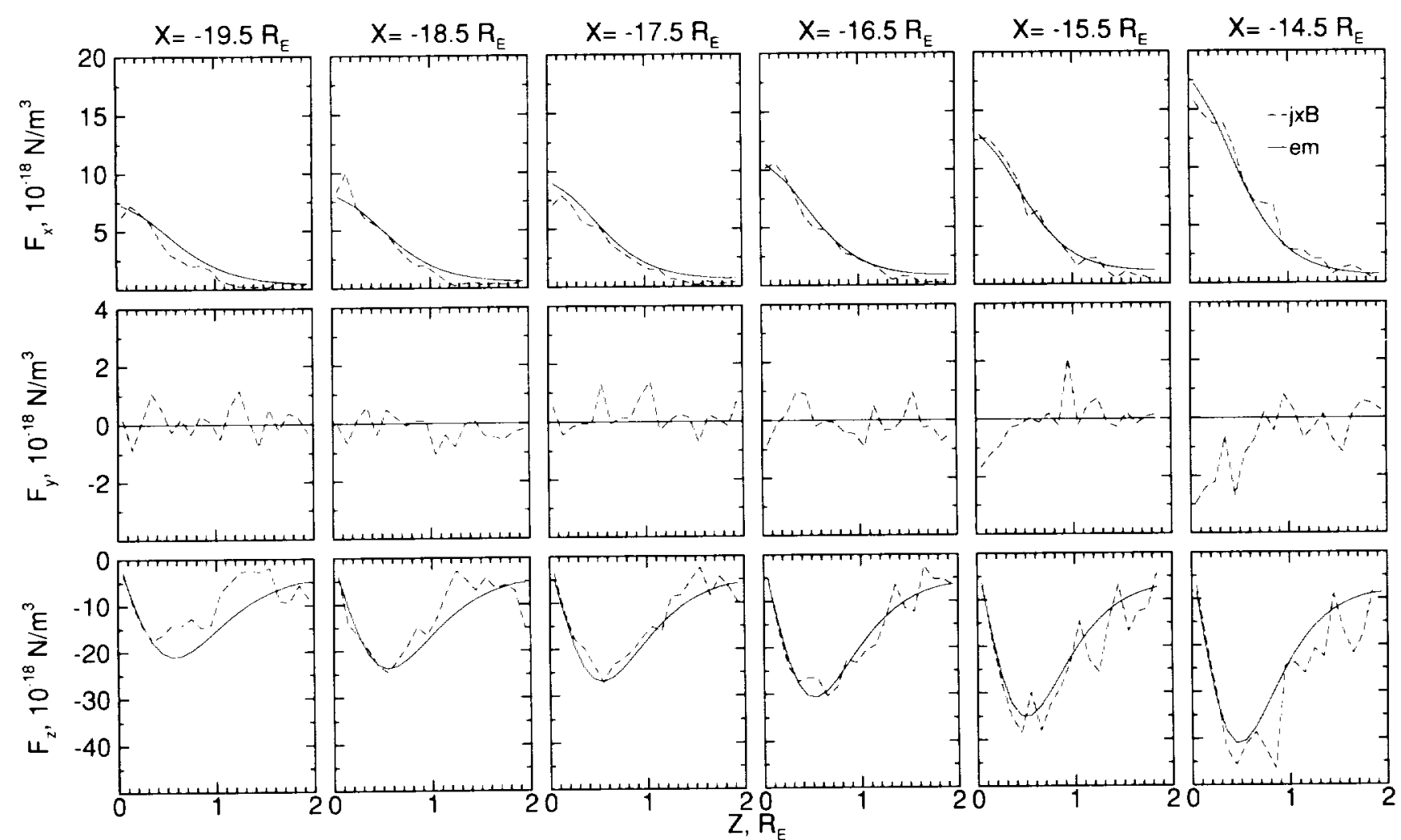

Figure 1. Each of the 18 panels shows a component of the electromagnetic (9) and $\mathbf{j} \times \mathbf{B}$ forces for a $1-R_{E}$ wide $\times$ box as a function of $z$. A comparison of (6) and (7) shows that these two forces should be equal in a consistent model.

The $y$ component of $\mathbf{j} \times \mathbf{B}$ in Figure 1 is produced only by $j_{x}$ and $j_{z}$. The least squares fitting routine did not consider $j_{x}$ or $j_{z}$ when selecting groups and calculating weighting factors. The particle to field energy density ratio or plasma $\beta$ is near 100 at the equator, where ions follow nonguiding center orbits. It is reassuring to see that even here the COT technique produces a model with reasonable balance between all components of the particle and field forces.

Figure 2 separates the electron and ion contributions to the $\mathbf{j} \times \mathbf{B}$ force. The $x$ and $z$ components of force from the two species are qualitatively similar, but ions clearly dominate. In contrast, the middle row of panels in Figure 2 shows that the $y$ components of the electron and ion forces both are large but opposite in direction so they nearly cancel when added together. The electron contribution is based on the smooth polynomial fit to pressures. The jagged ion contribution was calculated using the full ion distribution function. Noting that different scales are used for the three rows in Figure 1 , it is seen that the $y$ component of the total $\mathbf{j} \times \mathbf{B}$ force matches the desired $F_{y}^{E M}=0$ at least as well as the $x$ and $z$ components match the desired $F_{x}^{E M}$ and $F_{z}^{E M}$. If a model with significant $y$ dependence in the region of interest had been used, the $y$ component of the $\mathbf{j} \times \mathbf{B}$ and the electromagnetic field forces would be nonzero.

The reason why ions and electrons in our model have such large $y$ components of the $\mathbf{j} \times \mathbf{B}$ force in the sense shown (Figure 2) is that the plasma has a strong non-field-aligned earthward component of the bulk velocity even at $z=0$ (LK96). An average earthward drift has frequently been measured by satellites located near the neutral sheet [Baumjohann et al., 1989; Huang and Frank, 1994]. This cancellation of electron and ion $y$ forces is just what one would expect if the earthward bulk velocities are primarily $\mathbf{E} \times \mathbf{B}$ drift.
A real test of the balance between particle and electromagnetic forces involves comparing the right side of (7) or (9) with the left side of $(7)$. The solid lines in Figure 3 again are $F^{E M}$, which depends only on the preselected fields. The dashed lines in Figure 3 are the ion plus electron components of the particle forces, which depend only on the distribution functions. The calculated ion pressure force tends to be a little more jagged than the $\mathbf{j} \times \mathbf{B}$ terms in Figures 1 and 2 because the dominant contribution comes from taking numerical derivatives of the ion pressure tensor. Even so, errors between particle and field terms in Figure 3 are comparable to the errors associated with the selection of $j_{y}(x, z)$ as shown in Figure 1. This result is important for future work because any study using the energy equation depends heavily upon being able to evaluate the pressure tensor and other tensors with sufficient accuracy so that reliable derivatives can be calculated.

Electron and ion contributions to each term on the left side of (7) have been examined separately. Naturally $\partial / \partial t$ is zero in the steady state model. The ion contribution to the second term in (7) is only about $1 \%$ of the following terms for a quiet time model, and the electron contribution is even smaller. As discussed later, more rapid acceleration is needed to produce injection [DeForest and Mcllwain, 1971] and bursty bulk flow [Angelopoulos et al., 1992] events, so the first two terms are significant at times. Since electron pressures have been selected to be $1 / 7$ of the ion pressures, it is the divergence of the ion pressure tensor that is dominant on the left side of (7) and in the dashed curves of Figure 3.

\section{Substorm Effects}

Force concepts are used in this section to study substorms. Emphasis is placed on the unbalanced forces that are generated when current is disrupted. Substorm studies are complex because 


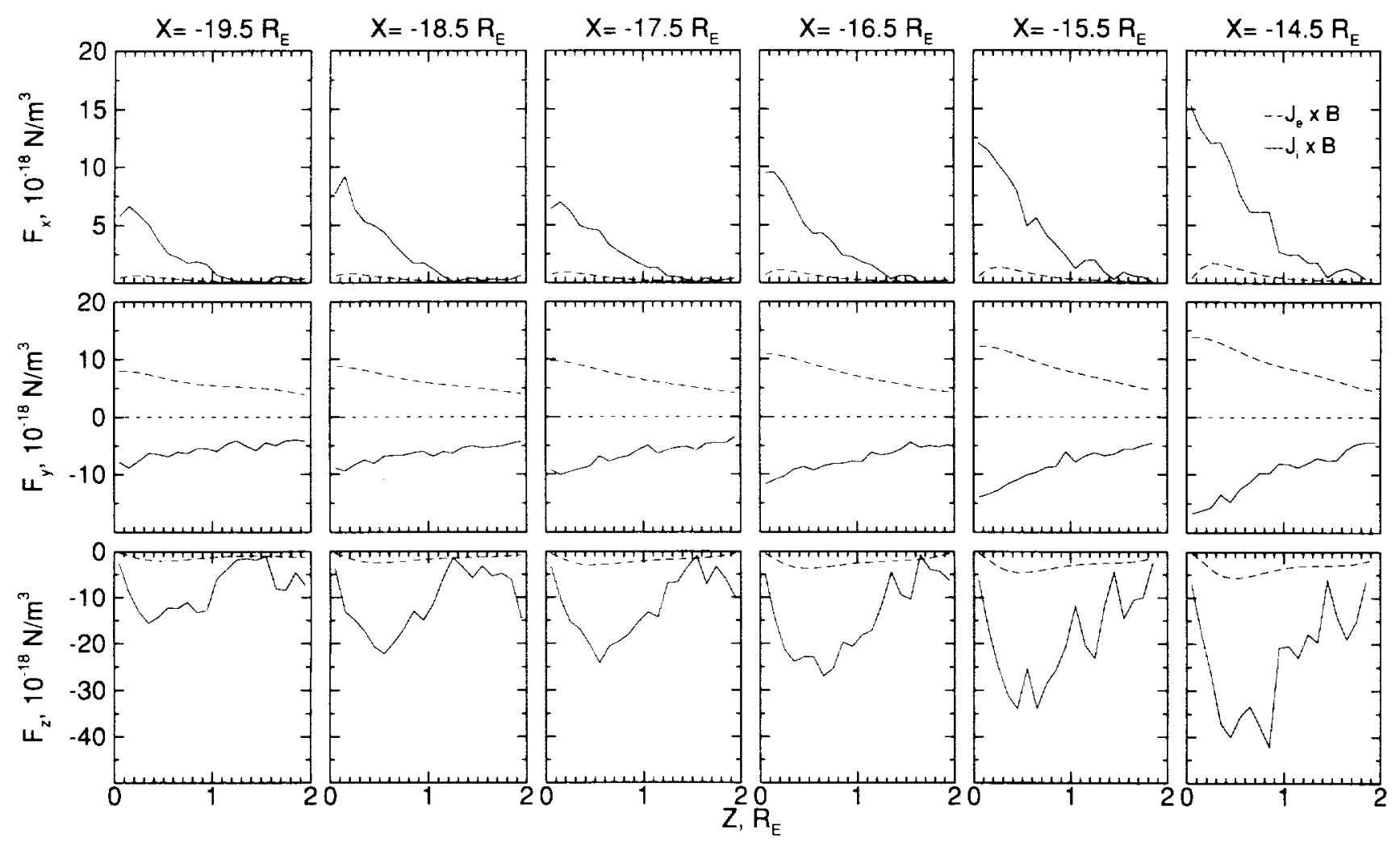

Figure 2. Electron and ion contributions to the $\mathbf{j} \times \mathbf{B}$ force are compared in the format that was used in Figure 1 .

many processes are interconnected. For example, plasma in the near-Earth injection region responds to changes in the fields that are produced by currents carried throughout the plasma sheet. Events such as substorm onsets, injections, and bursty bulk flows can be analyzed either in terms of instantaneous local forces and the associated plasma acceleration or from the perspective of current disruption and the evolution of polarization electric fields. The current system viewpoint is inherently global. It is hard to analyze the local substorm effects measured by a specific satellite using the current picture without introducing a model of the entire electrical circuit.

Although it is necessary to discuss currents in order to calculate forces, some satellite observations can be interpreted more directly from the perspective of forces. The $\mathbf{j} \times \mathbf{B}$ and $\boldsymbol{\nabla} \cdot \mathbf{P}$ terms in (6) give the forces that accelerate a unit volume of moving plasma. The local $\mathbf{j}$ and $\mathbf{B}$ can be measured by particle detectors and magnetometers, respectively, so that changes in the electromagnetic force term are directly observable. It is suggested here that the initial force imbalance is likely to be produced by a change in this electromagnetic term. This suggestion can be tested qualitatively by comparing sudden changes in the local plasma flow velocity to changes in the measured $\mathbf{j} \times \mathbf{B}$ force. The pressure force cannot be measured easily by a single satellite. However, it is likely that the principal changes in $\nabla \cdot \boldsymbol{P}$ follow the initial acceleration, taking place only after the plasma has moved and therefore become compressed and heated. One feature of the COT analysis is that fluid parameters are evaluated independently in each spatial box. It therefore is natural to consider plasma acceleration both in satellite data and in the COT analysis from this local perspective.

\subsection{Large-Scale Forces}

Siscoe [1966] calculated the force exerted by the Earth on the entire magnetotail by evaluating the $x$ gradient near the Earth of the fringing field produced by tail currents. Figure 4 is a sketch sum- marizing part of this basic work. The Earth is represented by a strong bar magnet with the south pole in the northern hemisphere. Each lobe of the magnetotail is treated as a D-shaped solenoid with current flowing in the plasma sheet and magnetopause. To illustrate forces within the magnetosphere, each lobe was treated as an object and replaced by an equivalent bar magnet in Figure 4. Considering only the three magnets, it is easy to see that each lobe magnet is attracted to the nearest pole of the Earth magnet. This illustrates the $x$ component of the electromagnetic force within the magnetosphere that keeps the magnetotail attached to the Earth in the presence of the tailward force exerted by the solar wind. Most of this earthward force is more commonly described in terms of field line tension. Attraction of the lobe magnets to each other represents the inward electromagnetic force in the $z$ direction that balances the outward plasma sheet particle pressure and keeps the high $\beta$ plasma sheet confined near $z=0$. An alternate description of $z$ forces is that the inward magnetic pressure exerted by the lobes balances the outward particle pressure exerted by the plasma sheet. The outward magnetic pressure exerted by the lobes on the magnetopause is balanced by the inward force exerted by the solar wind.

Since the Earth dipole field is fixed, any change in solar wind stress on the magnetotail requires a change in the current system and therefore a change in the strength and/or location of the equivalent lobe magnets. For example $K_{y}$, the sheet current density integrated through the thickness of the current sheet, increases and moves earthward during growth phase, implying that the solar wind exerts more force on the magnetotail [Siscoe and Cummings, 1969]. This is associated with an increase in the total magnetic flux in the tail and the resulting increases in tail flaring and $x$ momentum loss by the solar wind. The COT analysis only modeled a small region of the magnetotail so could not be used to calculate the total force on the entire magnetotail. Figures 1 and 3 show that the $z$ forces are about twice as large as the $x$ forces in our region of interest. 

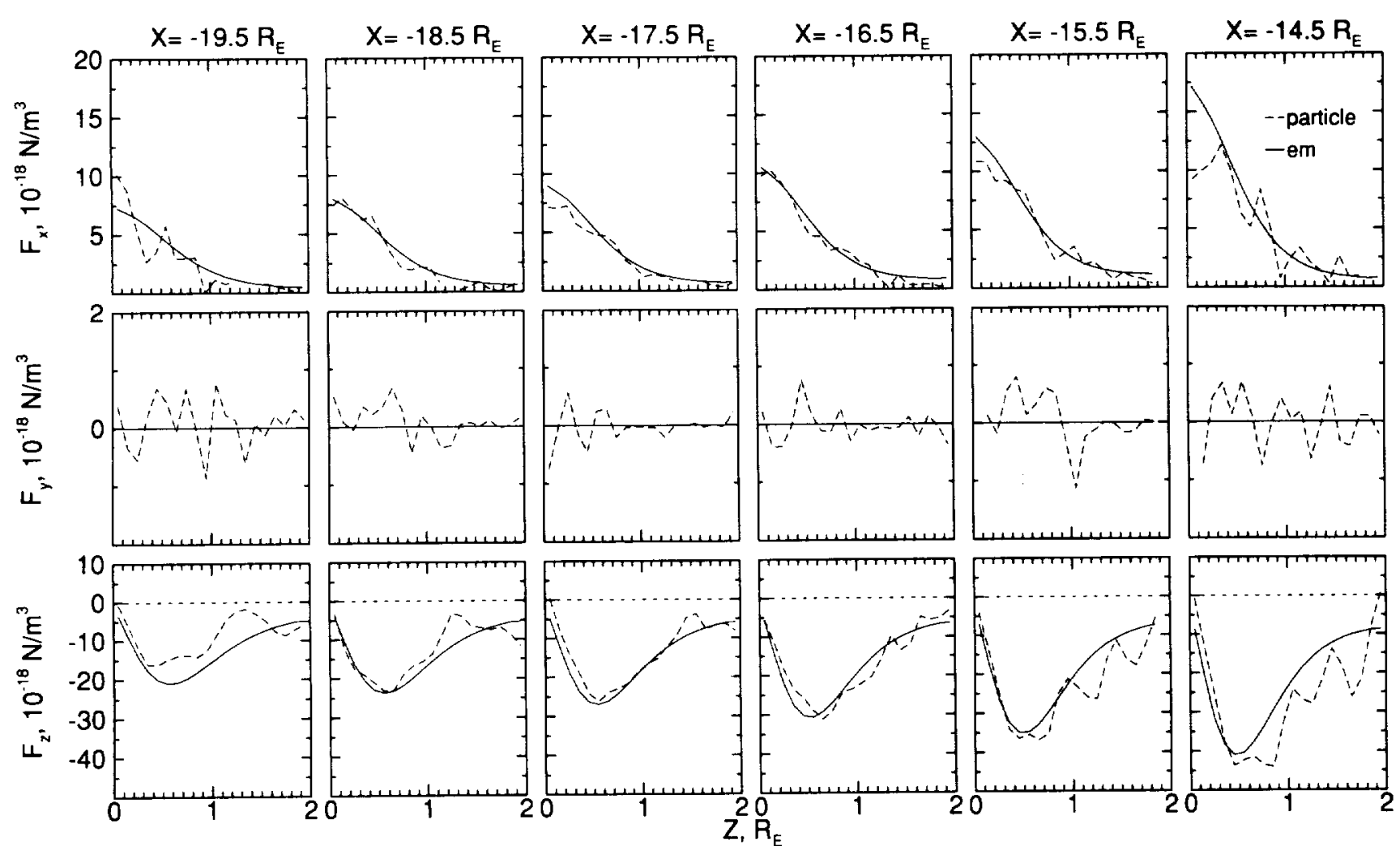

Figure 3. The two sides of (7) are compared. The electromagnetic force depends only upon the preselected fields and the particle force depends only upon the $f(\mathbf{r}, \mathbf{v})$ calculated during the COT analysis.

\subsection{Substorm Forces and Plasma Acceleration}

Most steady state studies of the quiet magnetotail neglect the net unbalanced force needed to accelerate plasma even though the plasma $\beta$ is near 100 at the center of the current sheet. $A$ comparison of terms in (6) shows the reason this omission is justified. A plasma element is accelerated earthward by the difference between the earthward force on a unit volume given by the $x$ component of $\mathbf{j} \times \mathbf{B}$ or $j_{y} B_{z}$ in the 2-D model and the tailward force provided by the $x$ component of $\nabla \cdot P$. Plots of the first two terms in (6) were not shown separately because the first is zero and the second is much smaller than the $\mathbf{j} \times \mathbf{B}$ and $\boldsymbol{\nabla} \cdot \mathbf{P}$ terms in the steady state COT model. LK 96 showed that $V_{x}$ near $z=0$ decreased by $50 \mathrm{~km} / \mathrm{s}$ as one moved earthward by $5 R_{E}$, yielding an average acceleration of $0.2 \mathrm{~km} / \mathrm{s}^{2}$. The net force needed for this weak quiet time acceleration in the COT model is $10^{-19} \mathrm{~N} / \mathrm{m}^{3}$, which represents only a $1 \%$ difference between the $\mathbf{j} \times \mathbf{B}$ and $\nabla$. $\mathbf{P}$ terms shown in Figure 3 .

If force imbalance and the associated acceleration always was as weak as in the quiet time COT model, it would take more than half an hour for an initially slowly moving plasma element to reach a speed of $400 \mathrm{~km} / \mathrm{s}$. During this time the plasma would move more than $60 R_{E}$. It is not known how long it takes for plasma in rapid flow regions to accelerate. Direct measurements of the instantaneous acceleration of a moving plasma element would be difficult to make even with an array of satellites. The mere observation of high speed flow also does not require a large local acceleration since no net force is needed to maintain even rapid steady flow. As an example, the net force on a rapidly moving mature plasma bubble can be zero [Chen and Wolf, 1993; Sergeev et al., 1996c]. However, injection events require strong plasma acceleration throughout the midnight region and any observed rapidly flowing plasma must have been accelerated somewhere. Accelerations and force imbalances well above those in the quiet time COT model therefore must sometimes be present in the magnetotail. Borovsky et al. [1997] emphasized that the observed instantaneous plasma velocities often fluctuate very rapidly in both magnitude and direction even during quiet times. This observation implies that large force imbalances may be common and therefore important to any study of the magnetotail.

As an example of the very rapid acceleration that may sometimes be present, it would take $40 \mathrm{~s}$ for an initially stationary plasma element to reach $400 \mathrm{~km} / \mathrm{s}$ if the average acceleration were $10 \mathrm{~km} / \mathrm{s}^{2}$. The plasma would move $1.3 R_{E}$ during this time. The force required for such an acceleration of the COT plasma is $F_{x}=$ $5 \times 10^{-18} \mathrm{~N} / \mathrm{m}^{3}$, which is a large fraction of the $\mathbf{j} \times \mathbf{B}$ and $\nabla \cdot P$ terms in Figure 3. We conclude that significant differences between $\nabla \cdot \mathbf{P}$ and $\mathbf{j} \times \mathbf{B}$ are needed if such large acceleration events take place either during substorms or during normal conditions in the middle magnetotail.

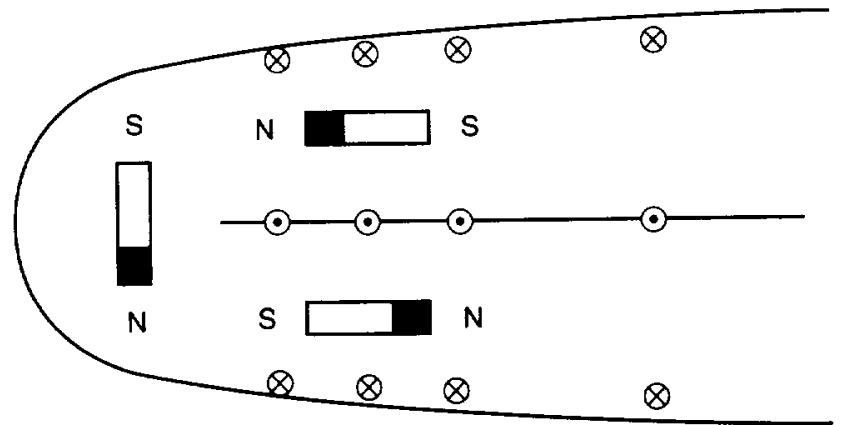

Figure 4. Sketch to illustrate the large-scale forces coupling the Earth and the magnetotail. 


\subsection{Substorm Currents}

In a model with little $y$ dependence, changes in $K_{y}$ extend from flank to flank and close over the northern and southern magnetopause. A sketch of the northern lobe current system is shown in Figure $5 \mathrm{a}$. This is the usual picture of growth phase. Observations suggest that $K_{y}$ initially is reduced at substorm onset only over a limited $(x, y)$ region, with the current loop closed by field aligned current to the ionosphere (Figure $5 \mathrm{c}$ ). The forces associated with this mode of current disruption are considered here.

The previous discussion shows that the earthward component of either $\mathbf{j} \times \mathbf{B}$ or $\nabla \cdot \mathbf{P}$ must change substantially to produce the plasma acceleration seen during substorms. Substorm onset usually is associated with a large change in magnetotail currents. This current change will produce changes in $\mathbf{B}$ throughout much of the plasma sheet. As suggested above, it therefore appears that changes in the $\mathbf{j} \times \mathbf{B}$ force should be examined in order to understand the cause of the initial substorm plasma acceleration.

Magnetic field changes have been used to estimate the location of a satellite with respect to the current disruption region [Lopez et al., 1988; Ohtani et al., 1992; Lui, 1996]. A reduction of $K_{y}$ in a given $(x, y)$ region results in a decrease of $B_{x}$ in the outer current sheet. A more important effect of a localized reduction of $K_{y}$ is the resulting increase of $B_{z}$ everywhere inside the diversion loop as shown in Figure 5d. The increase of $B_{z}$ in the inner magnetotail is a reliable observational signature of substorm onset. The assumption that $K_{y}$ decreases only in a localized region implies that $K_{y}$ initially remains constant while $B_{z}$ increases earthward of the disruption region. Although the shape of magnetic field lines will change everywhere, resulting in a change in the particle drift velocities, the average $K_{y}$ over an extended region cannot change greatly in a tail-like structure. A certain average tail lobe $B_{x}$ is needed so the outward lobe magnetic pressure balances the inward solar wind pressure at the magnetopause. A certain average $K_{y}$ is in turn needed to generate this lobe $B_{x}$. A large increase in $B_{z}$ combined with a relatively steady $K_{y}$ earthward of the disruption region therefore produces an increase of $K_{y} B_{z}$, the earthward force per unit area of the current sheet. At the same time $B_{z}$ decreases, the earthward force decreases, and field lines become more stretched outside a diversion loop (Figure 5d).

a)
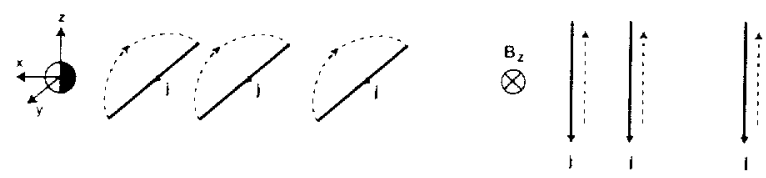

c)

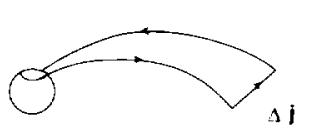

b)

$\mathrm{B}_{2}$

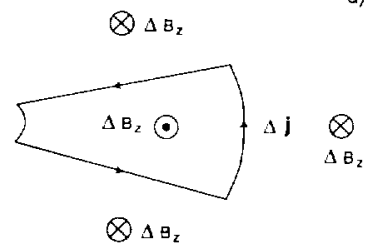

Figure 5. (a) Growth phase currents in the northern tail lobe, (b) view looking down on the equatorial plane of the growth phase currents and the resulting equatorial magnetic field, (c) perturbation currents that are added to those in Figure 5a at substorm onset, (d) view looking down on the equatorial plane of the substorm current loop and the resulting perturbation fields.
Pulkkinen et al. [1991] showed quantitatively how much the equatorial end points of field lines with fixed ionospheric foot points both inside and outside a current loop move, and Kaufmann [1987] showed how much foot points of field lines that connect to fixed tail locations move when current is diverted in a model magnetosphere. These two studies added extra current systems to existing magnetotail models. The nearly 2-D COT model that was used here is adequate to estimate the magnitude of the force changes needed to produce the observed substorm plasma acceleration near the center of a diversion loop. However, 3-D simulations or other time dependent techniques are needed for a comprehensive analysis of the current diversion process and of substorm injection events [Sanchez et al., 1993].

A reduction of $K_{y}$ through the formation of a diversion loop generates the important increase in $B_{z}$ more efficiently than would a decrease of $K_{y}$ from flank to flank with closure on the magnetopause. Figure $5 \mathrm{~d}$ shows that both the reduction of $K_{y}$ and the associated field aligned currents enhance $B_{z}$ everywhere inside a current diversion loop. In contrast, the east-west currents in the current sheet and magnetopause are in opposite directions when $K_{y}$ extends from flank to flank (Figure 5b). If this typical growth phase current system simply decreases, then $\Delta K_{y}$ in the current sheet increases $B_{z}$ and the change in the magnetopause part of the current loop decreases $B_{z}$ at the equator earthward of the current loop. The current sheet effect dominates at $z=0$ so that a small net increase of $B_{z}$ and the associated earthward force are produced in the earthward region at the equator by the fringing field of the full loop as the current in Figures $5 a$ and $5 b$ decreases.

A very simple current carrying wire model can make the above conclusions more quantitative. Earlier studies [Kaufmann, 1987; Kaufmann and Larson, 1989; Kaufmann et al. 1990; 1993] showed that reasonable estimates of magnetic field perturbations could be made with simple wire models provided the observer remained about one scale length away from the wires. The example used here models current disruption well out in the tail, but illustrates in general how sensitively $\Delta B_{z}$ depends on the current closure path. First, the Biot-Savart law was used with one of the loops shown in Figure 5a to calculate $\Delta B_{z}$ when the current is reduced from flank to flank and the loop closes around the semicircular magnetopause. The resulting $\Delta B_{z}$ is $\left(32^{-1 / 2}\right)\left(\mu_{o} \Delta I / \pi R\right) \equiv \Delta B_{o}$ as seen by an observer located at midnight in the equatorial plane at a distance $R$ either earthward or tailward of the localized disruption loop. In this expression $\Delta I$ is the current added to the wire loop and $R$ is the radius of the tail. Current disruption increases $B_{z}$ by $\Delta B_{0}$ at a distance $R$ earthward of the loop and decreases $B_{z}$ by $\Delta B_{o}$ at a distance $R$ tailward of the loop.

A simplified version of Figure 5d was used to make the comparison with current diversion to the ionosphere. A flat rectangular loop in the equatorial plane was used for the simple estimate instead of the circuit in Figure 5d which has current flowing along curved field lines. The rectangular model appears to be reasonable if disruption takes place in a thin plasma sheet. The contribution from the ionospheric leg of the rectangle was neglected because it was assumed to be far from the observer. The resulting increase in $\Delta B_{z}$ is $\left(1 / 2+2^{-1 / 2}\right)\left(\mu_{o} \Delta I / \pi R\right)=6.8 \Delta B_{o}$ when current disruption extends from flank to flank but the loop closes in the equatorial plane and the midnight observer is a distance $R$ earthward of the cross-tail disruption wire. If the current disruption rectangle is only half this wide, extending a distance $R / 2$ from midnight toward each flank, then the midnight observer located a distance $R$ earthward of the cross-tail leg sees $\Delta B_{z}$ increase by $\left(1+1.25^{1 / 2}\right)$ $\left(\mu_{o} \Delta I / \pi R\right)=12 \Delta B_{o}$. For an observer at midnight a distance $R$ 
tailward of the cross-tail leg, $B$, decreases by $1.2 \Delta B_{0}$ and 0.7 $\Delta B_{o}$ using the rectangles of half width $R$ and $R / 2$, respectively. We conclude that current diversion to the ionosphere can be an order of magnitude more efficient in producing $\Delta B_{z}$ and the associated earthward force on plasma inside the loop than a simple reduction of the growth phase current system. There is a much weaker dependence on the mode of disruption for plasma located tailward of the disruption region.

In summary, the principal magnetic signature of local dipolarization, the observation of a sudden increase in $B_{z}$, implies that a satellite is inside the principal diversion loop [Lopez et al., 1988; Ohtani et al., 1992; Lui, 1996]. This field change results in a net earthward force on a unit volume if $j_{y}(x, z)$ remains nearly constant at the observation point, i.e., if current is disrupted in a localized region tailward of the observer. On the other hand, a satellite that is just outside the diversion loop will see the magnetic field rapidly become more stretched. This is equivalent to a sudden enhancement of the growth phase perturbation, as noted by Ohtani et al. [1992]. Although the resulting acceleration of a moving element of plasma is difficult to measure, it should be possible to measure the change in $\mathbf{j} \times \mathbf{B}$ and therefore to calculate the expected initial acceleration at substorm onset.

\subsection{Pseudoexpansions and Multiple Expansions}

Several proposed disruption mechanisms require a thin current sheet. Such mechanisms can create new diversion events on the newly stretched field lines tailward of and at the sides of an original diversion loop. This sequence is similar to that seen during multiple expansion substorms [Rostoker, 1996; Sergeev et al., 1996b]. Figure 6 is a sketch of a possible multiple expansion process. It is assumed that the current increment labeled $\Delta \mathbf{j}_{1}$ is the first to be

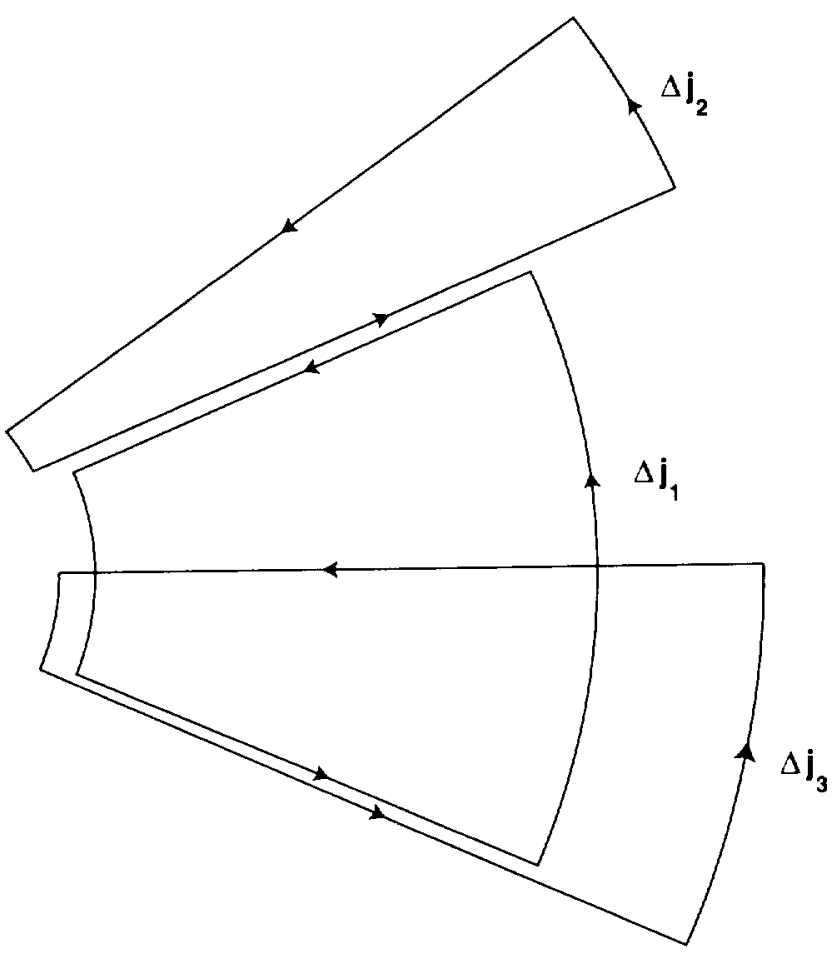

Figure 6. Growth of a multiple expansion substorm. Initiation of the first substorm current loop $\Delta \mathbf{j}_{1}$ causes a thinning of the plasma sheet everywhere outside this first loop. If nearby regions become thin enough, additional substorm loops $\Delta \mathbf{j}_{2}$ and $\Delta \mathbf{j}_{3}$ may be created. produced when an appropriate localized region of the tail becomes sufficiently thin. This current change causes regions adjacent to the initial diversion loop to become thinner (Figure 5d). A disruption process that requires a thin current sheet in an appropriate tail region then can generate the $\Delta \mathbf{j}_{2}$ and $\Delta \mathbf{j}_{3}$ diversion loops in Figure 6 , thereby extending the disruption region. A full substorm may require the formation of a sequence of diversion loops. A pseudoexpansion would result if only a small region of the tail becomes thin enough in a region that can cause disruption.

It was previously noted [Kaufmann, 1987] that nearly all ions remain in the magnetotail or outer magnetosphere during the brief period of substorm onset. A substantial fraction of the magnetotail electrons can be exchanged through field aligned currents with electrons in the ionosphere. However, if the total number of tail ions does not change suddenly at onset, then quasineutrality requires that the total number of electrons does not change either. Ions carry most of the cross-tail current in the COT current sheet model. Models containing very thin current sheets just before substorm onset [Lee et al., 1995; Pritchett and Coroniti, 1995; Ma et al., 1995; Birn et al., 1996] can have much of the cross-tail current carried by electrons. The above comments suggest that a study of substorm onset should consider mechanisms that reduce the cross-tail current carried by either species tailward of the injection region without decreasing the total number of particles in the magnetotail. Kaufmann et al. [this issue] describe such processes involving nonguiding center motion in a thin current sheet. Causes of the initial plasma sheet thinning also are discussed by Kaufmann et al. [this issue].

\section{Discussion and Summary}

A consistent orbit tracing (COT) method was used to find the particles needed to produce a quiet time model magnetotail. The model has little $y$ dependence and a characteristic thickness scale length of $0.7 R_{E}$ in the $-20 R_{E}<x<-14 R_{E}, 0<|z|<2 R_{E}$ region of interest. Orbit tracing started with an isotropic Maxwellian population of protons. Electrons were added using the guiding center approximations. Several similar nearly consistent current sheets were created in our earlier study (LK96) starting with monoenergetic protons at energies between 1.5 and $15 \mathrm{keV}$.

\subsection{Uniqueness}

Appendix $\mathrm{A}$ addresses some questions concerning the uniqueness of COT model results. It is concluded that the 2-D tail models used here are not uniquely generated by a specific distribution function. However, it was found that groups of nonguiding center ions following each type of orbit are required. The selection of particle energies for various runs was based on satellite observations. If only $5-\mathrm{keV}$ protons that mirror at low altitude (Speiser orbits) are present, then a current sheet is formed with a thickness of approximately $z_{o}=0.25 R_{E}$. The energy dependent parameter $z_{o}=$ $m v /\left[q B_{x y}\left(z_{o}\right)\right]$ is the point at which the particle's distance from the equatorial plane is equal to the $z$ component of the particle's local gyroradius. In the above expression $m, v$, and $q$ are the particle mass, velocity, and charge, and $B_{x y}^{2}=B_{x}^{2}+B_{y}^{2}$. The analysis showed that the addition of trapped particles can broaden the current sheet to a thickness of about $2 z_{0}$. Trapped particles meander back and forth across $z=0$ but never enter the region in which orbits spiral around field lines and particles are magnetically mirrored. The $2 z_{a}$ scale still is thinner than is required for the model in the $-20 R_{E}<x<-14 R_{E}$ region of interest. Particles on cucumber orbits that mirror throughout the current sheet also had to be 
included to produce the consistent current sheet. We were not able to generate a consistent quiet time model current sheet unless particles following all three orbit types were included. Therefore, although a unique $f(r, v)$ is not obtained, the mixture of particle reflection or mirror points is constrained if the particles are to carry the $j_{y}(x, z)$ that is needed to produce the model magnetic field. The more difficult question of uniqueness in a 3-D model with strong $y$ dependence is not addressed here.

Appendix B addresses another aspect of the question of uniqueness. The problem considered is how charge neutrality can be assured all along a field line on which electrons follow guiding center orbits and ions follow nonguiding center orbits. The COT method used isotropic electrons at the equator plus a parallel electric field to produce a neutral plasma. This method of introducing an $E_{||}$to maintain charge neutrality had been used in several early studies [Alfvén and Fälthammar, 1963, p. 162; Lemaire and Scherer, 1971; Chiu and Schulz, 1978]. Appendix B presents an alternate technique. An explicit method is developed to calculate the equatorial electron pitch angle distribution that will assure charge neutrality all along a field line with $E_{11}=0$ for a given variation of the ion density along the field line. We conclude that it is possible to pick a variety of electron distributions and electric fields in a charge neutral current sheet, so that neither $E_{\|}$nor the electron distribution function is uniquely specified by the selection of a magnetic field model.

\subsection{Force Balance}

The set of 120 distribution functions generated by the COT analysis was treated much as if one had data from a uniformly spaced array of 120 satellites. Evaluating the terms involving spatial derivatives in the momentum equation (6) requires a knowledge of fluid parameters throughout the region of interest. It therefore was easy to examine all the force and acceleration terms in the model calculations. In contrast, evaluating $\nabla \cdot \mathbf{P}$ and $(\mathbf{V} \cdot \nabla) \mathbf{V}$ are difficult when dealing with satellite data. The $\mathbf{j} \times \mathbf{B}$ and $\partial / \partial t$ terms in (6) are easier to measure because they require data at only one location.

Figures 1 to 3 were used to examine force balance. Since $j_{y}$ carried by the particles was considered when combining plasma groups, it was not surprising that the resulting $x$ and $z$ components of the $\mathbf{j} \times \mathbf{B}$ force were almost equal to the electromagnetic force required to create a consistent magnetotail (Figure 1). Even though $j_{x}$ and $j_{z}$ were not used in the fitting procedure, it was found that the resulting $y$ component of the $\mathbf{j} \times \mathbf{B}$ force also agreed with the requirements for consistency. Figure 2 showed that ion forces dominated the $x$ and $z$ components of $\mathbf{j} \times \mathbf{B}$, but electron and ion terms almost cancelled each other in the $y$ direction in the nearly 2-D modeling region. This cancellation was understood as primarily an effect of earthward drift which is associated with $E_{y}$. Figure 3 showed that the $\nabla \cdot \mathbf{P}$ force balanced the electromagnetic force to a good approximation in the model. This result demonstrated that the full pressure tensor is accurately calculated during a COT analysis. Inertial forces associated with the bulk motion of plasma particles were much smaller than the pressure forces in the quiet time model.

It is useful to compare the COT results to previous kinetic studies. The companion paper examines individual elements of the pressure tensor. Here we only consider the net forces. Burkhart et al. [1992] used a 1-D simulation. The ion source in this study was located far from $z=0$, so there were few trapped particles. Forces were found to be well balanced in thin current sheets with a small adiabaticity parameter $\kappa$. Force balanced solutions could not be obtained in thin current sheets when the average ion $\mathrm{K}$ approached one. Problems were encountered in balancing the $z$ component of particle and field forces. Our analysis required large contributions from ions on trapped and cucumber orbits in the thick quiet time current sheets associated with large K. Burkhart et al. [1992] noted that the force balance problem in thin large $\kappa$ current sheets may be associated with the nature of the 1-D model with few trapped ions. This issue is examined in more detail in the companion paper.

Ashour-Abdalla et al. [1993, 1994] used large-scale kinetic (LSK) orbit tracing to model all the magnetotail earthward of a distant neutral line. The magnetic field model was 2-D and based on T89. A drifting Maxwellian mantle source was used and orbits were traced for such a long time that Speiser, cucumber, and trapped orbits all became well populated before particles reached the middle magnetotail. Current sheets in this model tended to be thinner than is needed to produce the original T89 field. The thickness of the principal model current sheet was comparable to the thickness of the region in which trapped ions meander.

Pressure balance was examined in the LSK model. Forces were found to be reasonably well balanced, especially in almost 1-D regions of the tail. The force balance test used was a combination of the marginal firehose stability condition beyond the edge of the current sheet, which involves $x$ forces, and the need for $z$ forces to balance the lobe pressure. The guiding center approximations were well satisfied in the outer current sheet, so only the $P_{\| 1} / P_{\perp}$ ratio was needed in this test. Kaufmann et al., [this issue] show that other elements of $\boldsymbol{P}$ are needed for a detailed study of forces very close to $z=0$.

\subsection{Substorm Effects}

Substorm onset was studied as an application of the force balance analysis. The COT results pointed out that the average plasma acceleration in a quiet current sheet requires only small differences between the earthward $\mathbf{j} \times \mathbf{B}$ force and the tailward $\nabla . \mathbf{P}$ force but that bursty bulk flow and substorm injection events require more substantial force imbalances. Substorm onset involves a localized reduction of the integrated cross tail sheet current density $K_{y}$ near or somewhat tailward of that portion of the plasma sheet that begins to collapse. The reduction of $K_{y}$ must take place without significantly reducing the total number of particles in the tail during the brief onset period. Nonguiding center mechanisms that can do this are discussed by Kaufmann et al. [this issue]. The increase of $B_{z}$ and the associated increase in the earthward $K_{y} B_{z}$ force were treated as immediate causes of the initial plasma acceleration. It was found that diversion of cross-tail current along field lines to the ionosphere, which involves reduced $K_{y}$ in a region that is limited in both the $x$ and $y$ directions, is much more efficient at producing an increase in $B_{z}$ and the net earthward force than is a reduction of $K_{y}$ that extends from flank to flank and then closes on the magnetopause.

Cross-tail current disruption and the associated current diversion loop cause stretching of field lines to the east, west, and tailward of the diversion loop. If disruption is produced only in very thin current sheets Kaufmann et al. [this issue], then new diversion loops can be generated adjacent to the original loop. This mechanism provides one way to produce multiple expansions or the spreading of a substorm.

\section{Appendix A: Uniqueness}

One feature that the 2-D magnetostatic COT solutions have in common with electrostatic BGK [Bernstein et al., 1957] solutions 
is that neither method finds a unique distribution function. This appendix describes some of the simplest nonunique aspects of the analytic magnetic field model that was used. Nonunique aspects of the COT ion distribution functions and of the techniques used to treat electrons also are described.

\section{A 1.1. Harris-like Field Models}

The original 1-D Harris [1962] model and the many 2-D Harris-like variations $[K a n, 1973]$ start with an assumed $f(\mathbf{r}, \mathbf{v})$ at $z=$ 0 . All these models are based on the fact that any function that can be written in terms of only constants of the motion is a solution to the Vlasov equation. A drifting Maxwellian at $z=0$ was used for the 1-D and 2-D cases because it is easily written as a function of the hamiltonian $H$ and the $y$ component of the canonical momentum $P_{y}$, both of which are constants of the motion. Kan [1973] found that an infinite number of solutions for the 2-D magnetic field could be found with the assumed $f(\mathbf{r}, \mathbf{v})$, all of which satisfy Maxwell's equations. Three dimensional cases are more difficult because $P_{y}(\mathbf{r}, \mathbf{v})$ is not a constant of the motion. The uniqueness of 3-D analytic models is not considered here.

The Harris-like set of models was derived using some assumptions that are not realistic in the magnetotail. One assumption is that a reference frame exists in which $\mathbf{E}(\mathbf{r})=0$ everywhere. The assumption that most clearly disagrees with observations is that the electron and ion cross-tail bulk velocities $V_{e y}$ and $V_{i y}$ both are independent of $\mathbf{r}$ throughout the entire plasma sheet. The observations and COT results described in LK96 involved a $V_{i y}(r)$ that increases as one moves toward $z=0$ and toward the Earth. Finally, the Harris-like magnetotail model we used was based on an expansion in which certain small terms were assumed to be negligible [Birn et al., 1975; Zwingmann, 1983].

Perhaps the simplest way to see that the 2-D Harris-like fields can be generated by different distribution functions involves the dimensionless variables that are used to solve Ampere's law [Kan, 1973]. The distance $\mathbf{r}$ is normalized using the factor $h$ defined by $2 \mu_{o} h^{2}=T / n_{o} q^{2} V^{2}$ where $V=V_{i y}-V_{e y}, T=T_{i}+T_{e}$ is a thermal energy, and $n_{o}$ is a density normalization constant. The vector potential is normalized using $a=2 h\left(8 \pi n_{o} T\right)^{1 / 2}$. Two vector potentials $\mathbf{A}_{1}(x, z)$ and $\mathbf{A}_{2}(x, z)$ therefore are equal at the same unnormalized $(x, z)$ point if $n_{o 1} T_{1}=n_{o 2} T_{2}$ and $n_{o 1} V_{1}=$ $n_{o 2} V_{2}$ so that $h_{1}=h_{2}$ and $a_{1}=a_{2}$. For example, one could generate the same magnetic field by doubling the thermal energy and drift velocity of all ions and electrons while reducing the density by half since this does not alter either $\mathbf{r}$ or $\mathbf{A}(\mathbf{r})$. More important, the same $\mathbf{A}(x, z)$ can be produced by adding $n_{o 1} / 2$ of the particles discussed above with $T_{1}, V_{1}$ to $n_{o 2} / 2$ of a group with $T_{2}, V_{2}$. Similarly, this same $\mathbf{A}(x, z)$ can be produced by adding different amounts of many groups of drifting Maxwellians, each having the same $T / V$ ratio. A wide variety of distribution functions can be generated by combining various amounts of several drifting Maxwellians. Each $f(\mathbf{r}, \mathbf{v})$ produced in this manner generates the same 2-D Harris-like $\mathbf{B}(\mathbf{r})$.

\section{A 1.2. COT Results}

Rather than starting with a preselected $f(\mathbf{r}, \mathbf{v})$ and solving for $\mathbf{B}(\mathbf{r})$, the COT procedure started with a preselected $\mathbf{B}(\mathbf{r})$ and searched for $f(\mathbf{r}, \mathbf{v})$. A uniform cross-tail electric field was included for the case studied here. LK96 showed that satisfactory approximate solutions could be found for a given $\mathbf{B}(\mathbf{r})$ by starting with monoenergetic ions, even though the original Harris-like model was derived using a drifting Maxwellian at $z=0$. LK96 also found that particles with different energies could produce models that are nearly equal in approaching the goal of consistency. Figures 1 to 3 show that results of comparable consistency are obtained when the COT method is started using Maxwellian ions.

Although the COT method produced reasonable plasma sheets for nearly all 1-D and 2-D magnetic field models we have used to date, there are some models that cannot be produced using particles from a preselected parent population. For example, thin current sheets cannot be generated using ions that are so energetic that they cannot be deflected significantly by the magnetic field within the current sheet. As an extreme example, a model with a discontinuous $B$ could not be generated by any finite energy particles because the model would require an infinitely thin current sheet. The thinnest current sheets that can be produced by particles with a given energy have a thickness of approximately $z_{o}$. Kaufmann et al. [this issue] also show that we could not create thin current sheets using only particles with energies that produce the most chaotic orbits. Only limited tests have been carried out to date in an attempt to find particles that generate 3-D model fields with strong $y$ dependence. An extensive study of 3-D cases will be more challenging than the quiet time 2-D example used here. However, current in the actual magnetotail is carried by real ions and electrons. As a result, it should be possible to find a distribution of particles that will generate a sufficiently realistic model of the magnetotail.

With regard to the possibility of finding radically different particle distributions that produce the same magnetotail field, it was noted above that similar fields could be produced using a variety of quite different energy distributions. However, once the energy distribution is selected to agree with observations, then the COT studies found that the distribution of particle orbit types and reflection points was constrained. For example, we have traced many groups dominated by trapped particles and many groups dominated by Speiser particles. In every case the trapped particles show the characteristic negative $j_{y}$ near $z=0$ and positive $j_{y}$ in the $z_{o}<|z|<2 z_{0}$ region. Similarly, Speiser groups uniformly carry positive $j_{y}$ peaked at $|z|<z_{0}$. There always will be a range of particle distributions that produce almost equally good current sheets using the COT method. For example, it is well known [e.g., Stix, 1962, p. 114] that a Vlasov plasma can be approximated as closely as desired by combining many beams that follow free-streaming or unperturbed particle orbits. Highly structured distribution functions containing multiple beams have been proposed and observed in some parts of the plasma sheet [Frank et al., 1994; Ashour-Abdalla et al., 1996]. It is unlikely that the COT method will be able to select one specific highly structured distribution function from a group of structured distribution functions that produce similar fluid parameters.

\section{A1.3. Electron Assumptions}

Only ion orbits were traced in the COT analysis because thermal electrons obey the guiding center approximations in the model used here. If the magnetic moments of both ions and electrons were conserved, then steady state charge neutrality could be assured everywhere along a field line by selecting $E_{h}=0$ and the same pitch angle distribution for ions and electrons at the equator. However, the mirroring process will not assure plasma neutrality along a magnetotail field line when electrons follow guiding center orbits and ions follow nonguiding center orbits. Plasma was kept neutral along field lines in the COT calculations by using the Boltzmann relation to determine the required parallel electric field. Electrons usually were assumed to be isotropic and Maxwellian at $z=0$ [Kaufmann and $L u, 1993$ ]. The resulting small parallel electric field primarily modified the electron density to produce neutrality. 
The density of the more energetic ions was almost unaffected by this $E_{\mathrm{t}}$. A small relatively unimportant perpendicular electric field in the $x-z$ plane also was needed to keep $\nabla \times \mathbf{E}=0$ in this steady state 2-D tail model.

The above electron assumptions are not the only way to produce a neutral plasma. Appendix B describes a method of finding a nonisotropic pitch angle distribution for guiding center electrons that will assure plasma neutrality through simple mirroring effects with no parallel electric field. This technique was not applied in the COT runs carried out to date because the ion density was kept only in the rectangular region of interest near the equator. The ion density is needed all the way down to the Earth to use the technique in Appendix B. This appendix does show that the parallel electric field used in the analysis is not unique. A wide variety of electron pitch angle distributions could be combined with various $E_{\|}$ choices to maintain charge neutrality. However, very strong pitch angle dependence would be needed to cause electron densities to change as rapidly as do the ion densities near $z=1 R_{E}$ in Figure $10 \mathrm{a}$ of LK96 if this density change is to be produced by the mirror effect alone.

\section{Appendix B: Charge Neutrality}

This appendix shows how to find the pitch angle distribution of guiding center electrons that is needed for a plasma to be charge neutral everywhere along a magnetic field line. It is assumed that $E_{\|}=0$ and that the density of nonguiding center ions is known all the way from a reference point at which $B=B$, down to the Earth, where $n_{i}$ goes to zero. The reference point can be the equator in the middle magnetotail because electrons follow guiding center orbits all along the field lines. The variable $\xi=B_{1} / B$ is used to specify the location along a field line. The subscript 1 refers to the reference point where $\xi=1$. The electron magnetic moment must be conserved so that

$$
\sin ^{2} \theta / B=\sin ^{2} \theta_{1} / B_{1}
$$

where $\theta$ is the pitch angle. The region of interest is $\xi \leq 1$. The pitch angle distribution at the point $\xi$ is defined as

$$
F_{\xi}(\theta)=\int_{0}^{\infty} 2 \pi v^{2} f(\xi, v, \theta) d v
$$

where the velocity distribution function $f(\xi, v, \theta)$ is assumed to be independent of the phase angle around $B$. The particle number density is

$$
n(\xi)=2 \int_{0}^{\pi / 2} F_{\xi}(\theta) \sin \theta d \theta
$$

assuming symmetry about $\theta=\pi / 2$. Liouville's theorem

$$
F_{\xi}(\theta)=F_{1}\left(\theta_{1}\right)=F_{1}\left[\sin ^{-1}\left(\xi^{1 / 2} \sin \theta\right)\right]
$$

relates the distribution function at $\xi$ to the distribution function at the reference point. Substituting (B4) into (B3) and changing variables using (B1) gives

$$
n(\xi)=2 \int_{0}^{\sin ^{-1} \xi^{1 / 2}} F_{1}\left(\theta_{1}\right) \frac{\sin \theta_{1} \cos \theta_{1}}{\left[\xi\left(\xi-\sin ^{2} \theta_{1}\right)\right]^{1 / 2}} d \theta_{1}
$$

The purpose of this appendix is to invert (B5) to get the unknown pitch angle distribution $F_{1}\left(\theta_{1}\right)$ from $n(\xi)$, the known density distribution along a field line. The solution can be described most clearly by changing variables several times. First setting $\zeta=\sin ^{2} \theta_{1}$ gives

$$
\xi^{1 / 2} n(\xi) \equiv \phi(\xi)=\int_{0}^{\xi} \frac{F_{1}\left[\sin ^{-1}\left(\zeta^{1 / 2}\right)\right]}{(\xi-\zeta)^{1 / 2}} d \zeta
$$

Then multiplying both sides by $(\sigma-\xi)^{-1 / 2} d \xi$ where $\sigma$ is a new variable and integrating from 0 to $\sigma$ gives

$$
\begin{aligned}
& \int_{0}^{\sigma} \frac{\phi(\xi)}{(\sigma-\xi)^{1 / 2}} d \xi=\int_{0}^{\sigma} \frac{d \xi}{(\sigma-\xi)^{1 / 2}} \int_{0}^{\xi} \frac{F_{1}\left[\sin ^{-1}\left(\zeta^{1 / 2}\right)\right]}{(\xi-\zeta)^{1 / 2}} d \zeta \\
& =\int_{0}^{\sigma} F_{1}\left[\sin ^{-1}\left(\zeta^{1 / 2}\right)\right] d \zeta \int_{\zeta}^{\sigma} \frac{d \xi}{[(\sigma-\xi)(\xi-\zeta)]^{1 / 2}}
\end{aligned}
$$

where the order of integration has been reversed in the final form. This has been done because the $d \xi$ integral is just equal to $\pi$, as can be seen by changing variables from $\xi$ to $t=$ $(\xi-\zeta) /(\sigma-\zeta)$ where $\zeta$ is fixed for this integral. Equation (B7) then becomes

$$
\int_{0}^{\sigma} \frac{\phi(\xi)}{(\sigma-\xi)^{1 / 2}} d \xi=\pi \int_{0}^{\sigma} F_{1}\left[\sin ^{-1}\left(\zeta^{1 / 2}\right)\right] d \zeta
$$

The inversion is now completed by taking $d / d \sigma$ of both sides of (B8). A convenient form for the left hand side is obtained by changing variables from $\xi$ to $p=\sigma-\xi$ before taking the derivative. This gives

$$
\begin{gathered}
F_{1}\left[\sin ^{-1}\left(\sigma^{1 / 2}\right)\right]=\frac{1}{\pi} \int_{0}^{\sigma} \frac{\phi^{\prime}(\sigma-p)}{p^{1 / 2}} d p \\
=\frac{1}{2 \pi} \int_{0}^{\sigma} \frac{n(\xi)+2 \xi n^{\prime}(\xi)}{[\xi(\sigma-\xi)]^{1 / 2}} d \xi
\end{gathered}
$$

where $\phi(0)=0$, the primes indicate differentiation, and the last form has been transformed back from $\mathrm{p}$ to $\xi$. Setting $\sigma=$ $\sin ^{2} \theta_{1}$ gives the desired $F_{1}\left(\theta_{1}\right)$

$$
F_{1}\left(\theta_{1}\right)=\frac{1}{2 \pi} \int_{0}^{\sin ^{2} \theta_{1}} \frac{n(\xi)+2 \xi n^{\prime}(\xi)}{\left[\xi\left(\sin ^{2} \theta_{1}-\xi\right)\right]^{1 / 2}} d \xi
$$

A final form without the apparent problems at the limits can be obtained by changing variables from $\xi$ to $\alpha^{2}=\sigma-\xi$ in (B9) before setting $\sigma=\sin ^{2} \theta_{1}$

$$
F_{1}\left(\theta_{1}\right)=\frac{2}{\pi} \int_{0}^{\sin \theta_{1}} \phi^{\prime}\left(\sin ^{2} \theta_{1}-\alpha^{2}\right) d \alpha
$$

where the $\phi^{\prime}$ derivative is with respect to the argument $\sin ^{2} \theta_{1}-\alpha^{2}$. Equation (B11) can be evaluated for any $\theta_{1}$ provided the particle density $n\left(B_{1} / B\right)$ is known for all $B>B_{I}$. 
Acknowledgments. The reviewers made several suggestions that were incorporated into the final version of this paper. This material is based upon work supported by the National Science Foundation under grant ATM-94-22056 and by the National Aeronautics and Space Administration under grant NAGW-4539.

The Editor thanks two referees for their assistance in evaluating this paper.

\section{References}

Alekseyev, I. I., and A. P. Kropotkin, Interaction of energetic particles with the neutral sheet in the tail of the magnetosphere, Geomagn. Aeron., 10 , 615-619, 1970.

Alfvén, H., and C.-G. Fälthammar, Cosmical Electrodynamics, Oxford University Press, London, 1963.

Angelopoulos, V. W. Baumjohann, C. F. Kennel, F. V. Coroniti, M. G. Kivelson, R. Pellat, R. J. Walker, H. Lühr, and G. Paschmann, Bursty bulk flows in the inner central plasma sheet, J. Geophys. Res., 97. 4027-4039, 1992.

Ashour-Abdalla, M., J. P. Berchem, J. Büchner, and L. M. Zelenyi, Shaping of the magnetotail from the mantle: Global and local structuring, J. Geophys. Res., 98, 5651-5676, 1993.

Ashour-Abdalla, M., L. M. Zelenyi, V. Peroomian, and R. L. Richard, Consequences of magnetotail ion dynamics, J. Geophys. Res., 99. 14,891-14,916, 1994.

Ashour-Abdalla, M., L. A. Frank, W. R. Paterson, V. Peroomian, and L. M. Zelenyi, Proton velocity distributions in the magnetotail: Theory and observations, J. Geophys. Res., 101, 2587-2598, 1996.

Baumjohann, W., G. Paschmann, and C. A. Cattell, Average plasma properties in the central plasma sheet, J. Geophys. Res., 94, 6597-6606. 1989.

Bernstein, I. B., J. M. Greene, and M. D. Kruskal, Exact nonlinear plasma oscillations, Phys. Rev., 108, 546-550, 1957.

Birn, J., R. Sommer, and K. Schindler, Open and closed magnetospheric tail configurations and their stability, Astrophys. Space Sci., 35, 389-402, 1975.

Birn, J., M. Hesse, and K. Schindler, MHD simulations of magnetotail dynamics, J. Geophys. Res., 101, 12,939-13,954, 1996.

Borovsky, J. E., R. C. Elphic, H. O. Funsten, and M. F. Thomsen, The Earth's plasma sheet as a laboratory for flow turbulence in high- $\beta$ MHD. J. Plasma Phys., 57, 1-34, 1997.

Büchner, J., and L. M. Zelenyi, Deterministic chaos in the dynamics of charged particles near a magnetic field reversal, Phys. Lett. A, 118 , 395-399, 1986.

Büchner, J., and L. M. Zelenyi, Regular and chaotic charged particle motion in magnetotaillike field reversals. 1. Basic theory of trapped motion, J. Geophys. Res., 94, 11,821-11,842, 1989.

Burkhart. G. R., J. F. Drake, P. B. Dusenbery, and T. W. Speiser, A particle model for magnetotail neutral sheet equilibria, J. Geophys. Res., 97. $13,799-13,815,1992$.

Chen, J., and P. J. Palmadesso, Chaos and nonlinear dynamics of single-particle orbits in a magnetotaillike magnetic field, J. Geophys. Res., $91,1499-1508,1986$.

Chen, C. X., and R. A. Wolf, Interpretation of high-speed flows in the plasma sheet. J. Geophys. Res., 98, 21,409-21,419, 1993.

Chiu. Y., and M. Schulz, Self-consistent particle and parallel electrostatic field distributions in the magnetospheric-ionospheric auroral region, $J$. Geophys. Res., 83, 629-642, 1978.

Cole, G. H. A., and K. Schindler, On the equilibrium configuration of the geomagnetic tail. Cosmic Electrodyn., 3, 275-284, 1972.

Cowley, S. W. H., and R. Pellat, A note on adiabatic solutions of the one-dimensional current sheet problem, Planet. Space Sci.. 27, 265-271. 1979.

DeForest, S. E., and C. E. Mcllwain, Plasma clouds in the magnetosphere, J. Geophys. Res., 76, 3587-3611, 1971.

Eastwood, J. W., Some properties of the current sheet of the geomagnetic tail, Planet. Space Sci., 23, 1-14, 1975.

Erickson. G. M.. A quasi-static magnetospheric convection model in two dimensions, J. Geophys. Res., 97, 6505-6522, 1992.

Erickson, G. M., and R. A. Wolf, Is steady convection possible in the Earth's magnetotail?, Geophys. Res. Lett., 7, 897-900. 1980.

Francfort, P.. and R. Pellat, Magnetic merging in collisionless plasmas, Geophys. Res. Lett., 3, 433-436, 1976.

Frank, L. A., W. R. Paterson, and M. G. Kivelson, Observations of nonadiabatic acceleration of ions in Earth's magnetotail, J. Geophys. Res., 99, $14,877-14,890,1994$.
Gray, P. C., and L. C. Lee, Particle pitch angle diffusion due to nonadiabatic effects in the plasma sheet, J. Geophys. Res., 87, 7445-7452, 1982.

Harris, E. G., On a plasma sheath separating regions of oppositely directed magnetic field, Nuovo Cimento, 23, 115-121, 1962.

Hau, L.-N., Effects of steady state adiabatic convection on the configuration of the near-Earth plasma sheet, 2,J. Geophys. Res., 96, 5591-5596, 1991.

Huang, C. Y., and L. A. Frank, A statistical survey of the central plasma sheet, J. Geophys. Res., 99, 83-95, 1994.

Jackson, J. D., Classical Electrodynamics, John Wiley, New York, 1975.

Kan, J. R., On the structure of the magnetotail current sheet, J. Geophys. Res., 78, 3773-3781, 1973.

Kaufmann, R. L., Substorm currents: Growth phase and onset, J. Geophys. Res., 92, 7471-7486, 1987.

Kaufmann. R. L., and D. J. Larson, Electric field mapping and auroral Birkeland currents, J. Geophys. Res., 94, 15,307-15,319, 1989.

Kaufmann, R. L., and C. Lu, Cross-tail current: Resonant orbits, J. Geophys. Res., 98, 15,447-15,465, 1993.

Kaufmann, R. L., D. J. Larson, and C. Lu, Mapping and distortions of auroral structures in the quiet magnetosphere, J. Geophys. Res., 95, 7973-7994, 1990.

Kaufmann. R. L.. D. J. Larson, and C. Lu, Mapping and energization in the magnetotail, 2, Particle acceleration, J. Geophys. Res., 98, 9321-9333, 1993.

Kaufmann, R. L., C. Lu, and D. J. Larson, Cross-tail current, field-aligned current, and $B_{y}, J$. Geophys. Res., 99, 11,277-11,295, 1994.

Kaufmann, R. L.,' I. D. Kontodinas, B. M. Ball, and D. J. Larson, Nonguiding center motion and substorm effects in the magnetotail, J. Geophys. Res., this issue.

Larson, D. J., and R. L. Kaufmann, Structure of the magnetotail current sheet, J. Geophys. Res., 101, 21,447-21,461, 1996.

Lee, L. C., L. Zhang, G. S. Choe, and H. J. Cai, Formation of a very thin current sheet in the near-earth magnetotail and the explosive growth phase of substorms, Geophys. Res. Lett., 22, 1137-1140, 1995.

Lemaire, J., and M. Scherer, Simple model for an ion-exosphere in an open magnetic field, Phys. Fluids, 14, 1683-1694, 1971.

Lopez, R. E., D. G. Sibeck, A. T. Y. Lui, K. Takahashi, R. W. McEntire, T. A. Potemra, and D. Klumpar, Substorm variations in the magnitude of the magnetic field: AMPTE/CCE observations, J. Geophys. Res., 93, $14,444-14,452,1988$.

Lui, A. T. Y., Current disruption in the Earth's magnetosphere: Observations and models, $J$. Geophys. Res., I01, 13,067-13,088, 1996.

Ma, Z. W.. X. Wang, and A. Bhattacharjee, Growth, sudden enhancement. and relaxation of current sheets in the magnetotail: Two-dimensional substorm dynamics, Geophys. Res. Lett., 22, 2985-2988, 1995.

Ohtani, S., K. Takahashi, L. J. Zanetti, T. A. Potemra, R. W. McEntire, and T. lijima, Initial signatures of magnetic field and energetic particle fluxes at tail reconfiguration: Explosive growth phase, J. Geophys. Res., 97, 19.311-19.324, 1992.

Parker, E. N.. Newtonian development of the dynamical properties of ionized gases of low density, Phys. Re1., 107, 924-933, 1957.

Peroomian, V., and M. Ashour-Abdalla, Relative contribution of the solar wind and the auroral zone to near-Earth plasmas, in Cross-Scale Coupling in Space Plasmas, Geophys. Monogr. Ser., vol. 93, edited by J. L. Horwitz, N. Singh, and J. L. Burch, pp. 213-217, AGU, Washington, D. C., 1995.

Pritchett, P. L, and F. V. Coroniti, Formation of thin current sheets during plasma sheet convection, J. Geophys. Res., 100, 23,551-23,565, 1995.

Pulkkinen, T. I., H. E. J. Koskinen, and R. J. Pellinen, Mapping of auroral arcs during substorm growth phase, $J$. Geophys. Res.. 96, 21,087-21,094, 1991.

Rich. F. J., V. M. Vasyliunas, and R. A. Wolf, On the balance of stresses in the plasma sheet, $J$. Geophys. Res., 77, 4670-4676, 1972.

Rossi, B., and S. Olbert, Introduction to the Physics of Space, McGraw-Hill, New York, 1970.

Rostoker. G., Phenomenology and physics of magnetospheric substorms, $J$. Geophys. Res., 101, 12,955-12,973, 1996.

Sánchez, E. R., B. H. Mauk, and C.-I. Meng, Adiabatic vs. non-adiabatic particle distributions during convection surges, Geophys. Res. Lell., 20 , 177-180, 1993.

Sergeev, V. A., R. J. Pellinen, and T. I. Pulkkinen, Steady magnetospheric convection: A review of recent results, Space Sci. Rev., 75, 551-604. $1996 \mathrm{a}$.

Sergeev, V. A., T. I. Pulkkinen, and R. J. Pellinen, Coupled-mode scenario for the magnetospheric dynamics, J. Geophys. Res., 10I, 13,047-13,065, 1996b. 
Sergeev, V. A., V. Angelopoulos, J. T. Gosling. C. A. Cattell, and C. T. Russell, Detection of localized, plasma-depleted flux tubes or bubbles in the midtail plasma sheet, J. Geophys. Res., 101, 10,817-10,826, 1996c.

Siscoe, G. L., A unified treatment of magnetospheric dynamics with applications to magnetic storms, Planet. Space Sci., 14, 947-967, 1966.

Siscoe, G. L., and W. D. Cummings, On the cause of geomagnetic bays, Planet. Space Sci., 17, 1795-1802, 1969.

Sonnerup, B. U. O.., Adiabatic particle orbits in a magnetic null sheet, $J$. Geophys. Res., 76, 8211-8222, 1971.

Speiser, T. W., Particle trajectories in model current sheets, 1, Analytic solutions, J. Geophys. Res., 70, 4219-4226, 1965.

Speiser, T. W., Conductivity without collisions or noise, Planet. Space Sci., 18, 613-622, 1970.

Spitzer, L., Jr., Physics of Fully lonized Gases, pp. 155-159, John Wiley, New York, 1962.

Stix, T. H., The Theory of Plasma Waves, McGraw-Hill, New York, 1962.

Tsyganenko, N. A., A magnetospheric magnetic field model with a warped tail current sheet, Planet. Space Sci., 37, 5-20, 1989.
Wagner, J. S.. J. R. Kan, and S.-I. Akasofu, Particle dynamics in the plasma sheet, J. Geophys. Res., 84, 891-897, 1979.

Walker, R. J., T. Ogino, J. Raeder, and M. Ashour-Abdalla, A global magnetohydrodynamic simulation of the magnetosphere when the interplanetary magnetic field is southward: The onset of magnetotail reconnection, J. Geophys. Res., 98, 17,235-17,249, 1993.

Zwingmann, W., Self-consistent magnetotail theory: Equilibrium structures including arbitrary variation along the tail axis, $J$. Geophys. Res., 88, 9101-9108, 1983.

B. M. Ball, R. L. Kaufmann, I. D. Kontodinas, and D. J. Larson, Department of Physics, University of New Hampshire, Durham, NH 03824. (e-mail: dick.kaufmann@unh.edu)

(Received November 8, 1996; revised May 13, 1997; accepted June 11, 1997.) 